\title{
Assessment of Platelet Thrombus Formation under Flow Conditions in Adult Patients with COVID-19: An Observational Study
}

\author{
Stefano Ghirardello ${ }^{1}$ Anna Lecchi ${ }^{2}$ Andrea Artoni ${ }^{* 2}$ Mauro Panigada*3 Stefano Aliberti ${ }^{4,5}$ \\ Erica Scalambrino $^{2}$ Silvia La Marca ${ }^{2}$ Marco Boscarino ${ }^{2}$ Andrea Gramegna ${ }^{4}$ Paolo Properzi ${ }^{3}$ \\ Chiara Abruzzese $^{3}$ Francesco Blasi ${ }^{4,5}$ Giacomo Grasselli ${ }^{3,5}$ Fabio Mosca ${ }^{1,6}$ Armando Tripodi ${ }^{2}$ \\ Flora Peyvandi 2,5
}
${ }^{1}$ Neonatal Intensive Care Unit, Fondazione IRCCS Ca' Granda Ospedale Maggiore Policlinico, Milan, Italy
${ }^{2}$ Angelo Bianchi Bonomi Hemophilia and Thrombosis Center, Fondazione IRCCS Ca' Granda Ospedale Maggiore Policlinico, Milan, Italy
${ }^{3}$ Department of Anesthesia and Critical Care, Fondazione IRCCS Ca' Granda, Ospedale Maggiore, Milan, Italy
4 Respiratory Unit and Cystic Fibrosis Adult Center, Fondazione IRCCS
Ca' Granda Ospedale Maggiore Policlinico, Milan, Italy
${ }^{5}$ Department of Pathophysiology and Transplantation, Università degli Studi di Milano, Milan, Italy
${ }^{6}$ Department of Clinical Sciences and Community Health, Università degli Studi di Milano, Milan, Italy

\begin{abstract}
Address for correspondence Stefano Ghirardello, MD, Neonatal Intensive Care Unit, Department of Clinical Sciences and Community Health, Fondazione IRCCS Ca' Granda Ospedale Maggiore Policlinico, Milan, Italy (e-mail: stefano.ghirardello@mangiagalli.it).
\end{abstract}

Thromb Haemost 2021;121:1087-1096.

\begin{abstract}
Keywords

- platelets

- platelet function

- antiplatelet therapy

- arterial thrombosis

- blood coagulation

- T-TAS

- venous thromboembolism

Background Coronavirus disease 2019 (COVID-19) is associated with systemic inflammation, which may dysregulate platelet function. Total Thrombus-Formation Analysis System (T-TAS) is a flow-chamber device that analyses platelet-mediated thrombus formation in capillary channels through the following parameters: (1) the area under the flow-pressure curve (AUC), (2) occlusion start time (OST), time needed to reach OST, and (3) occlusion time (OT), time needed to reach the occlusion pressure. Methods and Findings Sixty-one COVID-19 patients admitted to intensive, subintensive, and low intensive care were prospectively enrolled according to the time of admission: group A (up to 8 days) $(n=18)$; group B (from 9 to 21 days) $(n=19)$, and group $C(>21$ days) $(n=24)$. T-TAS measurements were performed at enrolment and after 7 days. Median OST was similar among groups. AUC was lower in group A compared to $B(p=0.001)$ and $C(p=0.033)$. OT was longer in group $A$ compared to $B$ $(p=0.001)$ and $C(p=0.028)$. Platelet count (PC) was higher in group B compared to $A$ $(p=0.024)$. The linear regression showed that OT and AUC were independent from PC in group $A$ (OT: 0.149 [95\% confidence interval $[\mathrm{Cl}]:-0.326$ to 0.624$], p=0.513$ and AUC: 0.005 [95\% Cl: -0.008 to 0.017 ], $p=0,447)$. In contrast, in group $\mathrm{B}$, PC was associated with OT ( -0.019 [ -0.028 to 0.008$], p=0.023)$ and AUC (0.749 [0.358-1.139], $p=0,015$ ), similarly to group $C$. Conversely, patients with different illness severity had similar T-TAS parameters.
\end{abstract}

received

August 25, 2020

accepted after revision

December 3, 2020

published online

February 5, 2021
(C) 2021. Thieme. All rights reserved. Georg Thieme Verlag KG,

Rüdigerstraße 14,

70469 Stuttgart, Germany
DOI https://doi.org/

10.1055/s-0041-1722919.

ISSN 0340-6245. 
Conclusion COVID-19 patients display an impaired platelet thrombus formation in the early phase of the disease compared to later stages and controls, independently from illness severity.

\section{Introduction}

Coronavirus disease 2019 (COVID-19) is an acute multisystemic disease caused by severe acute respiratory syndrome coronavirus-2 (SARS-CoV-2) infection. The clinical manifestations in the most severe cases include bilateral interstitial pneumonia that, in approximately $5 \%$ of cases, evolves into acute respiratory failure with a high mortality rate. ${ }^{1,2}$ COVID-19 is associated with systemic inflammation, endothelial, and coagulation activation: hallmarks are elevated fibrinogen, von Willebrand factor (VWF), and D-dimers levels, ${ }^{3,4}$ contributing to hypercoagulability and increased mortality. ${ }^{5,6}$ The incidence of venous thromboembolism (VTE), including deep vein thrombosis (DVT) and pulmonary embolism (PE), varies from $6 \%$ to more than $30 \%$, irrespective of thromboprophylaxis. ${ }^{7-9}$ In the limited series of published autopsy studies, DVT was reported in up to 58\% of cases, while PE was the cause of death in one-third of deaths examined by Menter et al. ${ }^{10}$ In the absence of contraindications, prophylactic doses of low molecular weight heparin are recommended in all patients admitted for COVID-19 infection. ${ }^{11}$ The incidence of thrombocytopenia varies depending on reports; when present, it has been associated with increased mortality. ${ }^{1,12-14}$ Moreover, there is evidence that platelets and fibrin may locally aggregate and partially occlude small pulmonary arterial vessels and capillaries. $^{15}$

During inflammation due to viral and bacterial infections, platelets play a pivotal role in the coagulation process and the modulation of physiological and pathological responses to inflammation ${ }^{16-20}$; in some cases, enhanced or dysregulated platelet function might promote microvascular thrombosis. ${ }^{21,22}$ Indeed, some authors suggested that platelets could be a possible therapeutic target in COVID-19 patients. $^{23}$

The present study aimed to assess platelet function in COVID19 patients during different stages of the disease through the Total Thrombus-Formation Analysis System (T-TAS). This device allows real-time evaluation of platelet thrombus formation under controlled blood flow conditions. ${ }^{24,25}$

\section{Methods}

The study was carried out at the Fondazione IRCCS Ca' Granda Ospedale Maggiore Policlinico from April 30 to May 30, 2020. The Institutional Review Board (Comitato Etico Milano Area 2) approved the study protocol (n. 350_2020bis, April 24, 2020), and written informed consent was obtained from all patients. All procedures were carried out following the Helsinki Declaration.

\section{Study Design}

Prospective observational study recruiting adult patients affected by COVID-19 and admitted to the intensive care unit (ICU), subintensive, and low ICUs of our hospital.

The primary outcome of the study was to evaluate T-TAS parameters in relation to different stages of COVID-19.

Secondary outcomes were (1) to longitudinally evaluate the variations of T-TAS parameters at two different time points and (2) the association of T-TAS parameters with illness severity, mortality, and thrombotic events.

\section{Study Population}

Consecutive adult patients with COVID-19 were assessed for study eligibility.

Exclusion criteria included (1) presumed death in the next 24 hours, (2) use of antiplatelet and anti-inflammatory drugs in the previous seven days, and (3) hemorrhagic events in the previous 48 hours.

We collected demographic data, previous medical history, and therapies, and days from the onset of COVID-19 symptoms to hospital admission. At the time of blood sampling, we collected data on anticoagulant therapy, drugs administered for COVID-19 (antiretrovirals, hydroxychloroquine), ventilation support, and $\mathrm{PaO} 2 / \mathrm{FiO} 2$ ratio.

Patients were divided according to the interval between hospital admission and blood sampling as follows: (1) group A "early" patients, when admission occurred 8 days earlier or less from enrollment; (2) group B, “intermediate," when admission occurred from 9 to 21 days; and (3) group C, "late," when admission occurred more than 21 days before.

We included 32 healthy male and female volunteers, aged 25 to 62 years old, to serve as controls for T-TAS parameters, soluble P-selectin, CD40L, and whole blood cell count.

\section{Blood Collection and Measurements}

Blood was collected through arterial or venous lines in vacuum tubes containing $1 / 10$ volumes of trisodium citrate $0.109 \mathrm{M}$ (Becton Dickinson, Plymouth, United Kingdom). The sampling schedule was: T0 (at the time of enrollment)and T1 ( 7 days after the first blood sample). Laboratory investigations included: full blood cell count, ferritin, fibrinogen, activated partial thromboplastin time (aPTT), prothrombin time, D-dimer, VWF antigen, and ristocetin cofactor activity (VWF:Ag, VWF: RCo), factor VIII (FVIII) activity, P-selectin, and T-TAS.

T-TAS related platelet function:

To analyze flow-based thrombus formation, we used the modified T-TAS (Zacros, Fujimori Kogyo Co. Ltd., Tokyo, Japan), a semiautomated assay consisting of a tabletop instrument and disposable assay chip for the specific measurement of overall primary hemostatic function. ${ }^{22}$ The evaluation was performed 
with type 1 collagen-coated platelet chips (Nitta Gelatin, Osaka, Japan). Measurements were taken in a completed automated mode whereby blood samples were perfused into 26 capillaries path of the microchip with a precision pump. Briefly, whole blood samples $(350 \mu \mathrm{L})$ anticoagulated with Benzilsulfonil-D-Arg-Pro-4-amidinobenzilamide (BAPA anticoagulant) were applied to the PL chip at a flow rate of $24 \mu \mathrm{L} / \mathrm{min}$, which corresponds to a wall shear stress of $2,000 \mathrm{~s}^{-1}$ (approximate arteriolar shear stress). The generated thrombi, placed over the surface area coated with collagen, create a back pressure monitored by a pressure transducer in real time. The flow pressure curves were assessed for 10 minutes. The platelet thrombus formation on the chip's collagen surface was assessed using the following quantitative variables. The occlusion start time (OST), defined as the time (minutes) needed to reach the occlusion start pressure of $10 \mathrm{kPa}$ above the baseline pressure, is a measure of the platelet thrombus formation. The area under the flow pressure curve (AUC) below $60 \mathrm{kPa}$ for 10 minutes quantifies the thrombus stability (min*kPa). Finally, the time (minutes) at which the occlusion pressure $(60 \mathrm{kPa})$ is reached defines the occlusion time (OT) (for more details, see - Supplementary Fig. S1, available in the online version). T-TAS measurements were run in duplicate, and results were reported as the average value.

Soluble human P-selectin and soluble human CD40 ligand were measured in plasma samples from blood centrifuged for 20 minutes at $3,000 \times g$ using a commercially available enzyme-linked immunosorbent assay (Life Technologies Thermo Fisher Scientific, United Kingdom).

VWF:Ag and VWF:RCo were measured by immunoturbidimetric assays (Werfen, Orangeburg, New York, United States), and FVIII activity was measured by the one-stage clotting assay based on aPTT and FVIII deficient plasma (Werfen).

\section{Follow-Up}

Patients were evaluated daily for the occurrence of sepsis, hemorrhagic, and thrombotic events, which were recorded from enrollment to discharge or death.

\section{Statistical Analysis}

No published data on T-TAS parameters of COVID-19 patients were available when the study was designed, so the sample size was calculated assuming a within-group variance of OT (one of the T-TAS parameters to be evaluated for the primary outcome) of 3,000. With an alpha error of 0.05 and a power of 0.8 , the minimum detectable effect size (i.e., the square root of the ratio of the between-group variance and the withingroup variance) enrolling 15 to 20 patients per group would have been between 0.48 and 0.41 .

T-TAS parameters and platelet count were compared among different groups and with controls. A subsequent analysis was performed according to the patients' admission ward (ICU, subintensive, and low intensive care).

Data are presented as medians (min-max) for continuous variables and as counts and percentages for categorical variables. The nonparametric Kruskal-Wallis test was used to assess differences between groups. When a statistically signif- icant difference was observed, the Dunn test for multiple group comparison was employed. The Wilcoxon signed-rank test was used for longitudinal within-group comparisons. Linear regression analysis was used to determine the association between T-TAS parameters and platelets count in the three groups.

The Mann-Whitney test was performed to compare T-TAS and biochemical parameters (D-dimer, leucocytes, platelet count, FVII, VWF antigen, and VW:RCo) in survivors and notsurvivors and patients with and without thrombosis. We performed a receiver operating characteristic (ROC) analysis with $95 \%$ confidence interval $[\mathrm{CI}]$ to estimate the univariate logistic model's predictive capability between T-TAS parameters and mortality.

Correlations between hematocrit and T-TAS parameters were evaluated with the Pearson's correlation coefficient test.

$p$-Values are two-sided and correspond to a significance level of $<0.05$. Analyses were performed using the statistical software R, release 3.5.2 (R Foundation for Statistical Computing, Vienna, Austria).

\section{Results}

Between April and May 2020, we enrolled 61 patients divided as follow: 18 in group A, 19 in group B, and 24 in group C. Sixteen patients were admitted to low intensive care, 21 to the subintensive care, and 24 to ICU. - Table 1 summarizes the clinical characteristics of the cohort. The mean time between the onset of symptoms and hospital admission was 5.5 days (min-max 2-11 days). Demographic and clinical variables were not different among groups, except for the occurrence of VTE (19.6\% of the cohort) that was higher in groups B (4 patients) and C ( 8 patients) compared to group $A$ ( 0 patients) $(p=0.03)$. The laboratory characteristics of patients with and without VTE were similar, except for D-dimer that was significantly higher in patients with, compared to those without VTE (784 [100-6,078] vs. 2,250 [400-6,915] ug/L, $p=0.003$ ).

- Table 2 summarizes the results of laboratory tests and T-TAS parameters at T0. Platelet count, D-dimers, soluble P-selectin, and CD40L were significantly different among groups ( $p=0.002, p=0.025, p=0.009$, and $p=0.002$, respectively). At T0, the median OT was significantly longer in patients from group A compared to controls, groups B, and C. Median AUC was significantly lower in group A compared to controls, groups B, and C ( - Fig. 1). Median AUC and OT at T0 in group B were similar to controls. OST median values were similar among groups.

Soluble P-selectin and CD40L were similar among groups but were higher in groups B and C than controls; CD40L was higher in groups A and B than controls (-Fig. 1).

Linear regression showed that AUC and OT were independent from platelet count in group A (OT: IC95\% 0.149 [ -0.326 to 0.624 ], $p=0.513$ and AUC: IC95\% 0.005 [-0.008 to 0.017], $p=0.447$ ). In contrast, in groups B and C, OT and AUC values were dependent on platelet count. Specifically, in group B the model showed a significant relationship between platelet count and OT (IC95\% -0.019 [ -0.028 to -0.008$], p=0.023$ ) and AUC (0.749 [0.358-1.139], $p=0.015)$. Similar results 
Table 1 Clinical characteristics of the cohort

\begin{tabular}{|l|l|l|l|l|}
\hline & Cohort & Group A (early) & Group B (intermediate) & Group C (late) \\
\hline Patients (n) & 61 & 18 & 19 & 24 \\
\hline ICU & 24 & 5 & 7 & 12 \\
\hline Subintensive care & 21 & 7 & 6 & 8 \\
\hline Low intensive care & 16 & 6 & 6 & 4 \\
\hline Median age, y (range) & 61 & $57(29-94)$ & $66(39-95)$ & $60(39-81)$ \\
\hline Male \% & 63 & $72.2 \%$ & $47.4 \%$ & $70.8 \%$ \\
\hline BMI median (range) & $26(20-43)$ & $26(23-37)$ & $26(24-42)$ & $27(20-43)$ \\
\hline Diabetes (\%) & $9(14.7)$ & 3 & 4 & 2 \\
\hline Obesity (\%) & $25(40.9)$ & 9 & 7 & 9 \\
\hline Hypertension (\%) & $17(27.8)$ & 7 & 5 & 5 \\
\hline Cancer (\%) & $3(4.9)$ & 0 & 1 & 2 \\
\hline Other comorbidities (\%) & $36(59)$ & 15 & 14 & 7 \\
\hline PO FiO $_{2}$ median (range) & $233(57-483)$ & $253(150-414)$ & $230(57-367)$ & $270(133-483)$ \\
\hline Mechanical ventilation (\%) & $24(39.3)$ & 7 & 8 & 9 \\
\hline NCPAP/HFNC (\%) & $25(40.9)$ & 6 & 8 & 11 \\
\hline Oxygen therapy/SB (\%) & $12(19.6)$ & 5 & 3 & 4 \\
\hline Remdesivir (\%) & $5(8.1)$ & 2 & 3 & 0 \\
\hline Hydroxychloroquine (\%) & $17(27.8)$ & 5 & 8 & 4 \\
\hline Heparin (\%) & $40(65.5)$ & 9 & 13 & 18 \\
\hline VTE (\%) & $12(19.6)$ & 0 & 4 & 8 \\
\hline Bleeding events (\%) & $2(3.2)$ & 0 & 0 & 2 \\
\hline Deceased (\%) & $6(9.8)$ & 5 & 1 & 0 \\
\hline Time between admission and T0 (median d) & $17(2-59)$ & $5(2-8)$ & $15(9-19)$ & $31(22-59)$ \\
\hline
\end{tabular}

Abbreviations: BMI, body mass index; HFNC, high flow nasal cannula; ICU, intensive care unit; NCPAP, nasal continuous positive pressure; $\mathrm{PO}_{2}$, partial pressure oxygen; SB, spontaneous breathing; VTE, venous thromboembolism.

${ }^{\mathrm{a}} \mathrm{p}$-Value $=0.03$ (three-proportions $z$-test).

were found in group C (OT: IC95\% -0.015 [ -0.024 to -0.005$]$, $p=0.03$ and AUC [0.586 [0.199-0.973], $p=0.006$ ). Results were not influenced by VWF:Ag and VWF:RCo levels.

Six patients died, and 10 were discharged within 1 week from T0; 40 patients ( 8 in group A, 11 in group B, and 21 in group $\mathrm{C}$ ) were available for $\mathrm{T} 1$ sampling.

We observed a nonsignificant increase from T0 to T1 for both OT, AUC, platelet count, D-dimer, and P-selectin for patients in group A when longitudinally compared. Conversely, CD40L reduced between T0 and T1 ( - Table 3 ).

Mortality was significantly associated with lower platelet count (median 190 [60-306 $\left.\times 10^{\wedge} 9 / \mathrm{L}\right]$ vs. median $295\left[109-631 \times 10^{\wedge} 9 / \mathrm{L}\right], \quad p=0.034$ ), AUC (median 256 (108-304) vs. median 358 [13-500], $p=0.031$ ), and longer OT (median 11 [7.4-11] vs. median 5.7 [2.4-11], $p=0.006$ ).

ROC analysis with $95 \% \mathrm{Cl}$ showed that OT was useful for predicting mortality (AUC 0.858 ) at univariate analysis.

When patients were analyzed based on the intensity of care (ICU, subintensive care, and low intensive care), we did not find any difference in AUC and OT among groups (-Fig. 2).

However, AUC and OT from COVID-19 patients, independently from illness severity, were significantly different from controls. The platelet count of ICU patients was significantly higher compared to controls (-Fig. 2).

D-dimer and VWF antigen were significantly higher in the ICU than in low intensive care patients $(p=0.014$ and $p=0.005$, respectively).

P-selectin was higher in ICU and subintensive patients than controls; CD40L was higher than controls in all patients, independently from illness severity, but it reached statistical significance in patients admitted to low intensive and ICU only (-Fig. 2).

The Pearson's correlation coefficient test showed no correlation between hematocrit and AUC or OT (0.01 and 0.13, respectively). A weak direct correlation was present between OST and hematocrit $(0.3)$ in the whole cohort. Similar results were observed when the analysis was restricted to group A.

\section{Discussion}

The study showed that in COVID-19 patients, platelet thrombus formation assessed by T-TAS was impaired during the first week after hospital admission, compared to controls and patients at later disease stages. In contrast, we observed no 
Table 2 Laboratory tests and T-TAS parameters at T0

\begin{tabular}{|c|c|c|c|c|c|}
\hline Variables (reference range) & $\begin{array}{l}\text { Controls Median } \\
\text { (min-max) }\end{array}$ & $\begin{array}{l}\text { Group A (early) } \\
\text { Median } \\
\text { (min-max) }\end{array}$ & $\begin{array}{l}\text { Group B } \\
\text { (intermediate) } \\
\text { Median } \\
\text { (min-max) }\end{array}$ & $\begin{array}{l}\text { Group C (late) } \\
\text { Median } \\
(\min -\max )\end{array}$ & $p$-Value ${ }^{a}$ \\
\hline Serum ferritin (15-150 ug/L) & /I & $848(187-5,172)$ & $599(59-1,817)$ & $577(279-1712)$ & 0.678 \\
\hline G-reactive protein $(<0.5 \mathrm{mg} / \mathrm{dL})$ & /I & $1.3(0.4-10.3)$ & $5.8(0.1-18.6)$ & $5.2(0.0-17.2)$ & 0.750 \\
\hline Leucocytes $\left(10^{\wedge} 9 / \mathrm{L}\right)$ & $5.7(3.7-10.7)$ & $5.79(4.2-18.58)$ & $8.25(44.9-22.96)$ & $9.24(3.84-29.08)$ & 0.001 \\
\hline Platelet count $\left(10^{\wedge} 9 / \mathrm{L}\right)$ & $213(138-376)$ & $234(153-477)$ & $356(60-631)$ & 269 (109-629) & 0.002 \\
\hline Hematocrit (\%) & $35(31-43)$ & $37(28-51)$ & $29(25-35)$ & $27(22-36)$ & 0.001 \\
\hline PT-ratio (0.84-1.20) & /I & $1.14(0.91-1.31)$ & $1.12(0.93-1.28)$ & $1.08(0.89-1.29)$ & 0,359 \\
\hline aPTT-ratio (0.86-1.20) & /I & $1.05(0.90-2.39)$ & $1.13(0.77-2.14)$ & $0.99(0.76-1.22)$ & 0.302 \\
\hline Fibrinogen $(165-350 \mathrm{mg} / \mathrm{dL})$ & /I & $412(223-998)$ & $471(202-773)$ & 431 (153-929) & 0.878 \\
\hline D-dimer $[<500$ ug/L(FEU)] & /I & $456(100-6,078)$ & $1,047(360-2,761)$ & $1,158(200-6,915)$ & 0.025 \\
\hline VWF RCo (41-168\%) & /I & $327(103-478)$ & $300(134-549)$ & $402(207-523)$ & 0.068 \\
\hline VWF Ag ( 40-169\%) & /l & $401(115-598)$ & $357(169-1,090)$ & $435(259-689)$ & 0.227 \\
\hline FVIII (50-140\%) & /l & $224(124-386)$ & $278(186-573)$ & $290(218-475)$ & 0.067 \\
\hline OT (min) & $4.5(2.0-6.5)$ & $10.0(4.4-11.0)$ & $4.9(2.5-11.0)$ & $5.9(2.4-11.0)$ & 0.002 \\
\hline AUC (min*kPa) & $388(311-462)$ & $263(13-410)$ & $390(108-491)$ & $349(23-500)$ & 0.014 \\
\hline OST (min) & $1.6(1.1-2.6)$ & $2.1(1.1-11)$ & $1.8(1.1-3.9)$ & $2.0(1.1-8.4)$ & 0.909 \\
\hline Soluble P-selectin (ng/mL) & $56(30-101)$ & $63(43-153)$ & $81(39-155)$ & $75(42-151)$ & 0.009 \\
\hline Soluble CD40L (ng/mL) & $1.0(0.2-11.3)$ & $2.4(0.5-8.6)$ & $1.7(0.2-6.4)$ & $1.3(0.2-6.1)$ & 0.002 \\
\hline
\end{tabular}

Abbreviations: aPTT, activated partial thromboplastin time; AUC, area under the curve; FVIII, factor VIII; OST, occlusion start time; OT, occlusion time; PT, prothrombin time; T-TAS, Total Thrombus-Formation Analysis System; VWF Ag, von Willebrand factor antigen; VWF RCo, von Willebrand factor ristocetin cofactor activity.

${ }^{a}$ Kruskal-Wallis test.
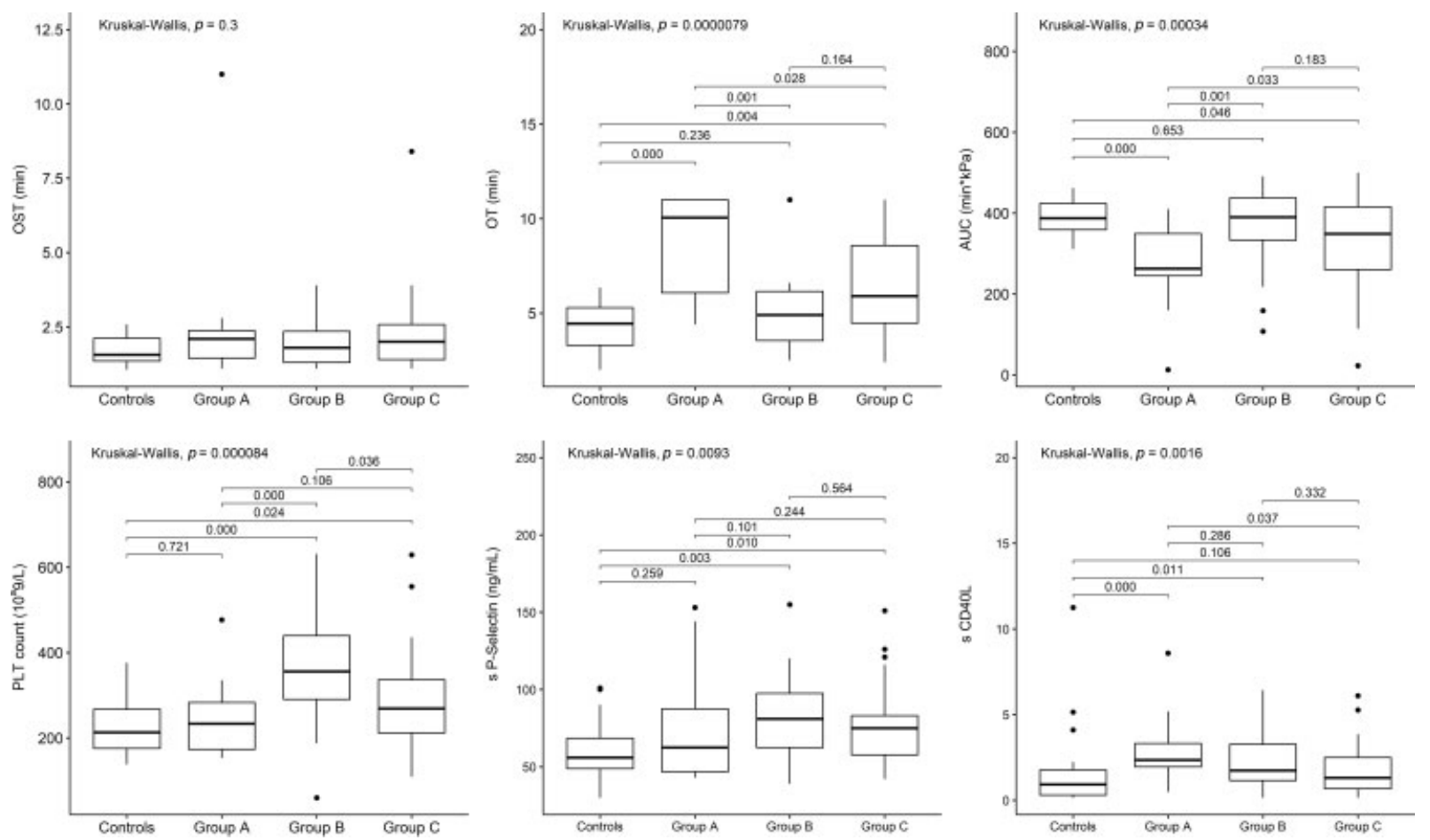

Fig. 1 Box-plot of Total Thrombus-Formation Analysis System (T-TAS) parameters, platelet count, soluble P-Selectin, and CD40L of patients at different disease stages (group A, B, and C) and healthy controls (Kruskal-Wallis and Dunn test). Occlusion start time (OST) was similar among groups and with healthy controls. Group A had longer median occlusion time (OT) compared to controls, group B, and C. Group A had a lower median area under the flow pressure curve (AUC) compared to controls, group B, and C. Platelet count was similar between controls and group A. Group B had significantly higher platelet count compared to controls and group A. Soluble P-selectin was higher in group B and C than controls. CD 40L was higher in group A and B, compared to controls. 
Table 3 Longitudinal evaluation of laboratory tests and T-TAS parameters

\begin{tabular}{|c|c|c|c|c|}
\hline Variables & & $\begin{array}{l}\text { Group A } \\
\text { Median (min-max) }\end{array}$ & $\begin{array}{l}\text { Group B } \\
\text { Median (min-max) }\end{array}$ & $\begin{array}{l}\text { Group C } \\
\text { Median (min-max) }\end{array}$ \\
\hline \multirow[t]{2}{*}{ Platelet count $\left(10^{\wedge} 9 / \mathrm{L}\right)$} & T0 & 251(153-477) & $340(188-631)$ & 285 (109-629) \\
\hline & $\mathrm{T} 1$ & $332(122-554)$ & $350(65-704)$ & $305(93-634)$ \\
\hline \multirow[t]{2}{*}{ D-dimer (ug/L) } & T0 & $609(268-1,030)$ & $948(572-2,250)$ & $1,160(200-6,820)^{a}$ \\
\hline & $\mathrm{T} 1$ & $902(251-12,000)$ & $1,060(676-1,690)$ & $936(200-6,160)^{a}$ \\
\hline \multirow[t]{2}{*}{ VWF:RCo (\%) } & T0 & $325(177-396)$ & $329(221-549)$ & $441(219-523)^{\mathrm{a}}$ \\
\hline & $\mathrm{T} 1$ & $325(113-483)$ & $265(168-555)$ & $387(152-485)^{\mathrm{a}}$ \\
\hline \multirow[t]{2}{*}{ VWF:Ag (\%) } & T0 & $390(234-527)$ & $362(261-1,090)$ & $460(259-689)$ \\
\hline & $\mathrm{T} 1$ & $392(130-670)$ & $385(217-1,010)$ & $451(193-610)$ \\
\hline \multirow[t]{2}{*}{ FVIII (\%) } & T0 & $234(186-369)$ & $300(203-573)^{a}$ & $295(218-475)$ \\
\hline & $\mathrm{T} 1$ & $239(131-333)$ & $239(171-436)^{\mathrm{a}}$ & $257(180-471)$ \\
\hline \multirow[t]{2}{*}{ OT (min) } & T0 & $9(5-11)$ & $5(3-11)$ & $6(2-11)$ \\
\hline & $\mathrm{T} 1$ & $7(4-11)$ & $7(2-11)$ & $6(3-11)$ \\
\hline \multirow[t]{2}{*}{ AUC $\left(\min ^{*} \mathrm{kPa}\right)$} & T0 & $290(160-410)$ & $406(159-491)$ & $349(23-500)$ \\
\hline & $\mathrm{T} 1$ & $364(191-451)$ & $367(108-510)$ & $350(20-451)$ \\
\hline \multirow[t]{2}{*}{ OST (min) } & T0 & $2(1-3)$ & $2(1-)$ & $2(1-8)$ \\
\hline & $\mathrm{T} 1$ & $1(1-3)$ & $2(1-5)$ & $2(1-11)$ \\
\hline \multirow[t]{2}{*}{ Soluble P-selectin (ng/mL) } & T0 & $64(49-144)$ & $88(39-155)$ & $70(42-151)$ \\
\hline & $\mathrm{T} 1$ & $87(34-150)$ & $72(38-135)$ & $77(41-138)$ \\
\hline \multirow{2}{*}{$\begin{array}{l}\text { Soluble CD40L } \\
(\mathrm{ng} / \mathrm{mL})\end{array}$} & T0 & $3.3(0.5-3.5)$ & $1.4(0.2-5.7)$ & $1.3(0.2-6.1)$ \\
\hline & $\mathrm{T} 1$ & $1.0(0.5-2.0]$ & $1.2(0.3-2.7)$ & $1.1(0.2-3.7)$ \\
\hline
\end{tabular}

Abbreviations: AUC, area under the curve; FVIII, factor VIII; OST, occlusion start time; OT, occlusion time; T-TAS, Total Thrombus-Formation Analysis System; VWF:Ag, von Willebrand factor antigen; VWF:RCo, von Willebrand factor ristocetin cofactor activity.

${ }^{a} p<0.05$ (Wilcoxon signed-rank test for paired samples) for comparison between T0 and T1.

difference in platelet thrombus formation among patients with different illness severity, although all patients, independently from the intensity care level, showed reduced platelet thrombogenicity compared to controls.

The platelet chip-based assay embedded in T-TAS explores the three major steps in platelet thrombus formation: the platelet adhesion mediated by VWF, the release of endogenous platelet agonists, and platelet aggregation.

The patients investigated showed adequate platelet adhesion at high shear rates, expressed by the OST, which at each disease stage considered in the study was not different from controls. The platelet adhesion mechanism on activated endothelium is mediated by the platelet GPIb-IX-V complex interaction with $\mathrm{VWF}^{26}$ which does not require functional platelets. Besides, such other adhesive proteins as platelets P-selectin glycoprotein ligand 1 , endothelial, and platelet P-selectin contribute to platelet adhesion. ${ }^{26,27}$

The preserved platelet adhesion to the subendothelial matrix, associated with normal-to-high VWF and fibrinogen concentrations in COVID-19 patients, may explain the lack of bleeding tendency in our cohort, despite a reduced platelet hemostatic function.

In contrast, the time required to occlude the capillaries (OT) and the AUC, both assessing platelet release and aggregation, varied with disease progression. In the early stage of the disease, the platelet thrombus formation showed poor stability, expressed by lower AUC and longer OT compared to the other groups and controls, independently from platelet count. According to platelet number variations at later stages of the disease, thrombus stability improved but remained impaired compared to controls.

Interestingly, patients with different degrees of illness severity showed a similar reduction in platelet thrombus formation than controls.

To further define platelet functions during SARS-CoV-2 infection, we measured soluble P-selectin and CD40L. Soluble P-selectin is a marker of endothelial cell and platelet activation that mediates the initial rolling of leukocytes onto the endothelium and activates monocytes to synthesize tissue factor. Because it is constitutively present in platelets and endothelial cells, increased soluble P-selectin may indicate endothelial cell dysfunction, platelet activation, or both. ${ }^{28}$ CD40L is a transmembrane protein of the tumor necrosis factor family located on the immune system's and vasculature cells, though $95 \%$ of the circulating CD40L is derived from platelets. ${ }^{29}$ Plateletassociated CD40L can initiate various inflammatory responses, including expression of inflammatory adhesion receptors, tissue factor expression, and chemokine release. ${ }^{29}$

We observed an opposite tendency in P-selectin and CD40L levels in relation to the progression of the disease; the former was increased while the latter was reduced as the disease progresses, both independently from illness severity. 

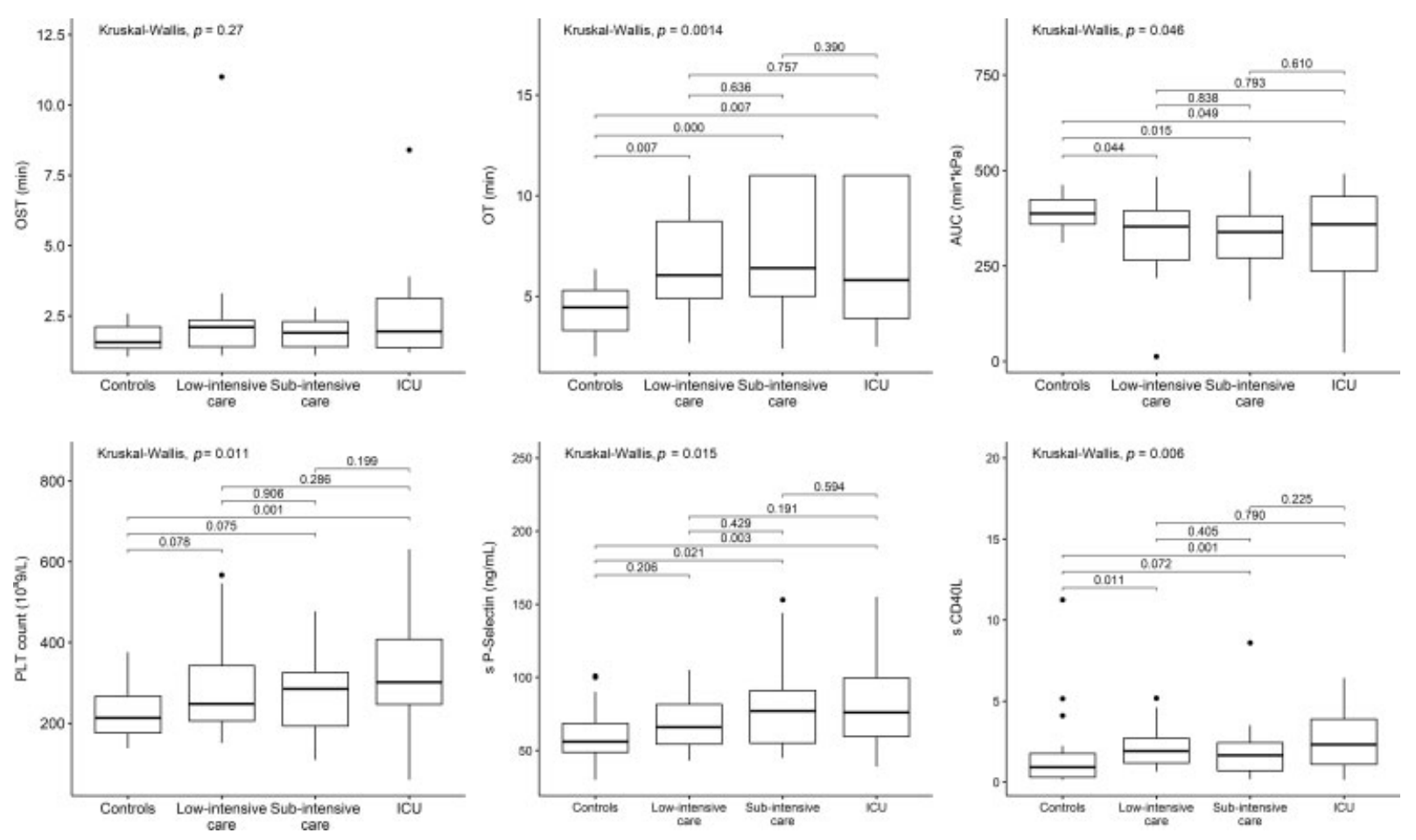

Fig. 2 Box-plot of Total Thrombus-Formation Analysis System (T-TAS) parameters, platelet count, soluble P-selectin, and CD40L of patients with different disease severity (intensive, subintensive, and low intensive care) (Kruskal-Wallis and Dunn test). T-TAS parameters, soluble P-selectin, and CD40L were similar among the three groups; area under the flow pressure curve (AUC) and occlusion time (OT) from COVID-19 patients were significantly different from controls independently from illness severity. Intensive care unit (ICU) patients showed a significantly higher platelet count compared to controls.

Indeed, CD40L concentration was significantly higher in the first weeks of hospitalization in patients than controls and showed a significant reduction over time. Conversely, patients with disease duration longer than 1 week (groups B and C) had P-selectin concentration higher than controls.

Recently, Goshua et al $^{30}$ reported that COVID-19 patients had higher CD40L and P-selectin concentration than controls, the first being independent from illness severity. In contrast, P-selectin was higher in the ICU than in non-ICU patients. The relatively unbalanced numbers of ICU versus less severe patients may explain P-selectin's differences between the two studies.

Previous studies ${ }^{31,32}$ suggested that platelets might contribute to the innate host's immune response during the SARS$\mathrm{CoV}-2$ infection. Manne et $\mathrm{al}^{31}$ reported altered platelet gene expression and functional response in patients infected with SARS-CoV-2. When compared to controls, COVID-19 patients presented with distinct features such as significantly increased P-selectin surface expression, upregulation of interferoninduced transmembrane protein 3 (which is an antiviral immune protein), increased circulating platelet-neutrophil, monocyte, and T-cell aggregates, which were all independent from the severity of illness.

Zaid et $\mathrm{al}^{32}$ showed that in COVID-19 patients, platelets are hyperactivated and release granules contents independently from disease severity and that platelet adhesiveness was increased at low shear rate flowing conditions. Interestingly, both studies ${ }^{31,32}$ detected SARS-CoV-2 messenger ribonucleic acid in platelets from both nonsevere and severe COVID-19 patients.
The initial response to the SARS-CoV-2 generally occurs in the first week after infection and is characterized by the activation of macrophages, monocytes natural killer (NK) cells, and cytokines production. ${ }^{33}$ Platelets contribute to activate innate immunity and modulate host immune response to bacterial and viral infections through interaction with the immune effector cells. Indeed, during H1N1 virus infection, platelets release $\alpha$ granule content after virus internalization, ${ }^{34}$ promoting platelet-neutrophil interaction mediated by P-selectin and CD40L, which are essential to initiate the innate immune response. ${ }^{20,35}$

Based on our results, we hypothesize that platelets exert an immunological role during the first stage of COVID-19, at the expense of their hemostatic functions. The sustained platelet stimulation and degranulation during the onset of virus replication may downregulate platelet hemostatic function and stimulate platelet immune response. ${ }^{36-38}$ Accordingly, reduced functions and biochemical signs of exhaustion have been reported in NK and CD8 + T cells during COVID-19. ${ }^{16}$

The lack of correlation between platelet count, OT, and AUC, during the initial stage of the disease, may support the hypothesis that platelets undergo a process of granules content depletion that leads to exhaustion of their hemostatic capacity. Accordingly, we observed that CD40L concentration, mainly derived from platelets, ${ }^{29}$ was the highest at the disease's early stage. In the subsequent stages of the disease, T-TAS parameters as well as platelet count start to improve, although the latter was more evident with AUC, which quantifies the thrombus stability. The progressive recovery of hemostatic 
function observed in groups B and C, besides the reduction of circulating CD40L, may be related to newly released fullfunctional platelets.

Moreover, in group A, the longitudinal evaluation showed increasing platelet count, P-selectin, and platelet thrombus formation and reduced CD40L concentration 1 week after the first sampling, reaching values similar to those observed in patients sampled during the intermediate disease phase.

Despite the lack of statistical significance, these observations are consistent with the hypothesis that the platelet function in COVID-19 recovers with disease progression and suggest a different origin of circulating P-selectin and CD40L in SARS-CoV-2 infection. CD40L increases at the early stage of the disease when platelets are involved in the immune response, while P-selectin increases later, possibly as a consequence of endothelial dysregulation.

We confirmed the previously described association between lower platelet count and mortality. ${ }^{1,12-14}$ Similarly, platelet functions were significantly reduced in deceased patients than survivors, and OT was highly predictive of death. However, due to the relatively small number of patients, these findings require further investigation. As previously shown, ${ }^{14,39}$ D-dimer was higher in nonsurvivors than survivors, although it did not reach statistical significance.

Our cohort's VTE occurrence rate amounted to $20 \%$, with no differences related to platelet function and number, and standard coagulation parameters, except for D-dimers that were significantly higher in patients with than without VTE. All patients, irrespective of the disease stage, had VWF (antigen and activity) and FVIII higher than controls, without association with thrombotic events, as previously reported. ${ }^{30,40}$

There are strengths and limitations of the study that should be recognized.

Strengths are the investigation of primary hemostasis in COVID-19 patients performed with a relatively simple bedside device designed to assess the overall platelet function under flow conditions, which mimics much more than the measurement of other individual parameters the overall platelet function.

Among the limitations, it should be recognized that we did not assess for specific activation or aggregation defects. The latter would have supported our findings but would have required specific and time-consuming procedures, which, although available in the laboratory, were difficult to perform owing to the time constraints and sample volumes limitation imposed by the study.

Furthermore, the surmised immunological role of platelets during SARS-CoV-2 infection, although plausible, has not been investigated. Indeed, whether our findings are uniquely associated with SARS-CoV-2 infection or other viral illnesses might show similar platelet function changes require further investigations.

Besides, we enrolled patients based on admission time, but this time point may not reflect disease duration, as the onset of symptoms may vary among patients.
Finally, laboratory tests were not performed for all consecutive patients admitted to the three hospital wards, and therefore, we cannot exclude selection bias.

\section{Conclusion}

The study indicates that platelets' ability to promote thrombus formation when assessed in vitro by a device that operates under flow conditions mimicking those operating in vivo is reduced in COVID-19 patients, only in the early phase of the disease, and is independent of illness severity. We also provide evidence that platelet activation/dysfunction involves both severe and nonsevere forms of COVID-19. The findings point to the possible role of antiplatelet drugs in patients with COVID-19 and pave the way for an ongoing clinical trial (ClinicalTrials.gov: NCT04365309) aimed to assess their role in this condition.

\section{What is known about this topic?}

- COVID-19 is associated with a state of hypercoagulability with elevated fibrinogen, D-dimers, and Von Willebrand that increase the risk of arterial and venous thrombosis.

- Evidence from autopsies showed that platelets and fibrin might locally contribute to occlude small pulmonary arterial vessels and capillaries.

- Platelet function under controlled blood flow conditions has been poorly investigated in COVID-19 patients.

\section{What does this paper add?}

- In COVID-19, platelet thrombus formation assessed by the real-time device T-TAS was reduced during the first week after hospital admission, compared to patients at later disease stages.

- Patients with different illness severity showed similar platelet thrombus formation that was significantly reduced compared to controls.

\section{Authors' Contributions}

S.G. conceptualized and designed the study, contributed to data analysis, interpretation, and drafted the article. A.L. contributed substantially to data acquisition, analysis, interpretation, and revised it critically for important intellectual content. A.A. and M.P. contributed to analysis, interpretation, and critically revised the manuscript for important intellectual content. S.A., E.S., M.B., and S.L.M. contributed substantially to data acquisition, analysis, and interpretation of data. A.G., P.P., and C.A. contributed to the analysis and interpretation of data. F.B., G.G., F.M., A.T., and F.P. contributed to the conception and design of the study, data interpretation, and revised the article for important intellectual content. All authors gave final approval of the version to be submitted and any revised version. 


\section{Funding}

This work was (partially) supported by the Italian Ministry of Health - Bando Ricerca Corrente.

\section{Conflict of Interest}

None declared.

\section{Acknowledgment}

We would like to thank Luigi Ghilardini for his contribution in creating the figures of the manuscript.

\section{References}

1 Huang C, Wang Y, Li X, et al. Clinical features of patients infected with 2019 novel coronavirus in Wuhan, China. Lancet 2020;395 (10223):497-506

2 Guan WJ, Ni ZY, Hu Y, et al;China Medical Treatment Expert Group for Covid-19. Clinical characteristics of coronavirus disease 2019 in China. N Engl J Med 2020;382(18):1708-1720

3 Soy M, Keser G, Atagündüz P, Tabak F, Atagündüz I, Kayhan S. Cytokine storm in COVID-19: pathogenesis and overview of antiinflammatory agents used in treatment. Clin Rheumatol 2020;39 (07):2085-2094

4 Varga Z, Flammer AJ, Steiger P, et al. Endothelial cell infection and endotheliitis in COVID-19. Lancet 2020;395(10234):1417-1418

5 Tang N, Li D, Wang X, Sun Z. Abnormal coagulation parameters are associated with poor prognosis in patients with novel coronavirus pneumonia. J Thromb Haemost 2020;18(04):844-847

6 Zhou F, Yu T, Du R, et al. Clinical course and risk factors for mortality of adult inpatients with COVID-19 in Wuhan, China: a retrospective cohort study. Lancet 2020;395(10229):1054-1062

7 Al-Samkari H, Karp Leaf RS, Dzik WH, et al. COVID-19 and coagulation: bleeding and thrombotic manifestations of SARSCoV-2 infection. Blood 2020;136(04):489-500

8 Helms J, Tacquard C, Severac F, et al;CRICS TRIGGERSEP Group (Clinical Research in Intensive Care and Sepsis Trial Group for Global Evaluation and Research in Sepsis) High risk of thrombosis in patients with severe SARS-CoV-2 infection: a multicenter prospective cohort study. Intensive Care Med 2020;46(06):1089-1098

9 Llitjos JF, Leclerc M, Chochois C, et al. High incidence of venous thromboembolic events in anticoagulated severe COVID-19 patients. J Thromb Haemost 2020;18(07):1743-1746

10 Menter T, Haslbauer JD, Nienhold R, et al. Postmortem examination of COVID-19 patients reveals diffuse alveolar damage with severe capillary congestion and variegated findings in lungs and other organs suggesting vascular dysfunction. Histopathology 2020;77(02):198-209

11 Bikdeli B, Madhavan MV, Jimenez D, et al;Global COVID-19 Thrombosis Collaborative Group, Endorsed by the ISTH, NATF, ESVM, and the IUA, Supported by the ESC Working Group on Pulmonary Circulation and Right Ventricular Function. COVID-19 and thrombotic or thromboembolic disease: implications for prevention, antithrombotic therapy, and follow-up. J Am Coll Cardiol 2020;75(23):2950-2973

12 Lippi G, Plebani M, Henry BM. Thrombocytopenia is associated with severe coronavirus disease 2019 (COVID-19) infections: a meta-analysis. Clin Chim Acta 2020;506:145-148

13 Zhao X, Wang K, Zuo P, et al. Early decrease in blood platelet count is associated with poor prognosis in COVID-19 patients-indications for predictive, preventive, and personalized medical approach. EPMA J 2020;11:1-7

14 Zhang A, Leng Y, Zhang Y, et al. Meta-analysis of coagulation parameters associated with disease severity and poor prognosis of COVID-19. Int J Infect Dis 2020;100:441-448

15 Fox SE, Akmatbekov A, Harbert JL, Li G, Brown JQ Vander Heide RS. Pulmonary and cardiac pathology in Covid-19: the first autopsy series from New Orleans. Lancet Respir Med 2020. Doi: 10.1101/2020.04.06.20050575

16 Ren YR, Golding A, Sorbello A, et al. A comprehensive updated review on SARS-CoV-2 and COVID-19. J Clin Pharmacol 2020;60 (08):954-975

17 Koupenova M, Clancy L, Corkrey HA, Freedman JE. Circulating platelets as mediators of immunity, inflammation, and thrombosis. Circ Res 2018;122(02):337-351

18 Nicolai L, Massberg S. Platelets as key players in inflammation and infection. Curr Opin Hematol 2020;27(01):34-40

19 Morrell CN, Pariser DN, Hilt ZT, Vega Ocasio D. The platelet Napoleon complex-small cells, but big immune regulatory functions. Annu Rev Immunol 2019;37:125-144

20 Jackson SP, Darbousset R, Schoenwaelder SM. Thromboinflammation: challenges of therapeutically targeting coagulation and other host defense mechanisms. Blood 2019;133(09):906-918

21 Guo L, Rondina MT. The era of thromboinflammation: platelets are dynamic sensors and effector cells during infectious diseases. Front Immunol 2019;10:2204

22 Hottz ED, Bozza FA, Bozza PT. Platelets in immune response to virus and immunopathology of viral infections. Front Med (Lausanne) 2018;5:121

23 Qiu J, Ma J, Zhang S, Han J, Liu S. Promoting platelets is a therapeutic option to combat severe viral infection of the lung. Blood Adv 2020;4(08):1640-1642

24 Hosokawa K, Ohnishi T, Kondo T, et al. A novel automated microchip flow-chamber system to quantitatively evaluate thrombus formation and antithrombotic agents under blood flow conditions. J Thromb Haemost 2011;9(10):2029-2037

25 Kaikita K, Hosokawa K, Dahlen JR, Tsujita K. Total ThrombusFormation Analysis System (T-TAS): clinical application of quantitative analysis of thrombus formation in cardiovascular disease. Thromb Haemost 2019;119(10):1554-1562

26 Moroi M, Jung SM, Shinmyozu K, Tomiyama Y, Ordinas A, DiazRicart M. Analysis of platelet adhesion to a collagen-coated surface under flow conditions: the involvement of glycoprotein VI in the platelet adhesion. Blood 1996;88(06):2081-2092

27 Coenen DM, Mastenbroek TG, Cosemans JMEM. Platelet interaction with activated endothelium: mechanistic insights from microfluidics. Blood 2017;130(26):2819-2828

28 Lorant DE, Topham MK, Whatley RE, et al. Inflammatory roles of Pselectin. J Clin Invest 1993;92(02):559-570

29 André P, Nannizzi-Alaimo L, Prasad SK, Phillips DR. Plateletderived CD40L: the switch-hitting player of cardiovascular disease. Circulation 2002;106(08):896-899

30 Goshua G, Pine AB, Meizlish ML, et al. Endotheliopathy in COVID19-associated coagulopathy: evidence from a single-centre, cross-sectional study. Lancet Haematol 2020;7(08):e575-e582

31 Manne BK, Denorme F, Middleton EA, et al. Platelet gene expression and function in patients with COVID-19. Blood 2020;136 (11):1317-1329

32 Zaid Y, Puhm F, Allaeys I, et al. Platelets can associate with SARSCov-2 RNA and are hyperactivated in COVID-19. Circ Res 2020. Doi: 10.1161/CIRCRESAHA.120.317703

33 Azkur AK, Akdis M, Azkur D, et al. Immune response to SARS-CoV2 and mechanisms of immunopathological changes in COVID-19. Allergy 2020;75(07):1564-1581

34 Koupenova M, Corkrey HA, Vitseva O, et al. The role of platelets in mediating a response to human influenza infection. Nat Commun 2019;10(01):1780

35 Nicolai L, Gaertner F, Massberg S. Platelets in host defense: experimental and clinical insights. Trends Immunol 2019;40 (10):922-938

36 Heijnen $\mathrm{H}$, van der Sluijs P. Platelet secretory behaviour: as diverse as the granules ... or not? J Thromb Haemost 2015;13(12): 2141-2151

37 Etulain J, Negrotto S, Carestia A, et al. Acidosis downregulates platelet haemostatic functions and promotes neutrophil 
1096 Platelet Function in Adult COVID-19 Patients: An Observational Study Ghirardello et al.

proinflammatory responses mediated by platelets. Thromb Haemost 2012;107(01):99-110

38 Pareti FI, Capitanio A, Mannucci L, Ponticelli C, Mannucci PM. Acquired dysfunction due to the circulation of "exhausted" platelets. Am J Med 1980;69(02):235-240

39 Gungor B, Atici A, Baycan OF, et al. Elevated D-dimer levels on admission are associated with severity and increased risk of mortality in COVID-19: a systematic review and meta-analysis. Am J Emerg Med 2021;39:173-179

40 Grobler C, Maphumulo SC, Grobbelaar LM, et al. Covid-19: the rollercoaster of fibrin(ogen), D-dimer, Von Willebrand factor, Pselectin and their interactions with endothelial cells, platelets and erythrocytes. Int J Mol Sci 2020;21(14):5168 